Online Journal of Biological Sciences 7 (1): 8-11, 2007

ISSN 1608-4217

(C) 2007 Science Publications

\title{
Relationship Between Blood Fibrinogen Concentration and Pathological Features of Cancer Patients: A 139-case Clinical Study
}

\author{
${ }^{1}$ Da-Yong Lu, ${ }^{2}$ Xue-Liang Chen, ${ }^{3}$ Min Huang, ${ }^{3}$ Bin Xu and ${ }^{3}$ Jian Ding \\ ${ }^{1}$ School of Life Sciences, Shanghai University, Shanghai, PR China \\ ${ }^{2}$ Department of Oncology and Thermo-therapy, Central Hospital of Jing-An District, Shanghai, PR China \\ ${ }^{3}$ Shanghai Institute of Materia Medica, Chinese Academy of Sciences, Shanghai, PR China
}

\begin{abstract}
Angiogenesis and coagulation are among the most consistent host responses to the presence of a malignant tissue. Pathological angiogenesis and coagulation are often occurred in patients with solid tumors, especially in the occurrence of neoplasm metastasis and as targets for anti-metastatic drugs such as antiangiogenesis agents, coagulation-mediated agents and anticancer drugs. Since fibrinogen (Fib) is the most abundant and key haemostatic protein taking part in angiogenesis and coagulation, its biological and pathophysiological roles in cancer patients are intriguing. To continue foundational and translational research on Fib-related cancer pathogenesis, a phase II survey of 139 patients was carried out at the Central Hospital of Jing-An district and Shanghai University, Shanghai, China. The mean BFC of the cancer patients in this survey was overall about $35-50 \%$ greater than that in the normal population. This study showed that the mean BFC was higher in patients with long-distance metastases $\left(\mathrm{N}_{1} \mathrm{M}_{1}\right.$ patients) than in patients with no sign of long-distance metastases $\left(\mathrm{N}_{0} \mathrm{M}_{0}\right.$ patients). Mean BFCs were $4.42 \mathrm{~g} / \mathrm{L}(\mathrm{n}=21)$ in patients with lung cancer, $4.36 \mathrm{~g} / \mathrm{L}$, and in patients with hepatic cancer $(n=5)$, and $4.63 \mathrm{~g} / \mathrm{L}$ in patients with stomach cancer $(n=8)$, all higher than the average value of the cancer patients overall $(4.16 \mathrm{~g} / \mathrm{L})$. However, patients with bowel and colon cancers $3.79 \mathrm{~g} / \mathrm{L}(\mathrm{n}=16)$ showed lower than them. BFC levels increased with increasing cancer duration (latency $>1$ year). There was a slight decrease in BFC after one or two treatment cycles, but a more marked decrease after surgery. We propose that the BFC level in cancer patients may be influenced by and related to many aspects of cancer progression such as metastatic conditions, tumor origins, patient's pathological stage and disease latency. As an important first-hand pathologic-therapeutics relationship study, it provides evidence for the potentiality of a new approach of Fib-targeted as individualized cancer chemotherapy and decides the stages such therapeutics may be best applied.
\end{abstract}

Key words: Fibrinogen, neoplasm, metastasis, cancer chemotherapy, cancer pathogenesis

\section{INTRODUCTION}

Fibrinogen (Fib), a major clotting component of the blood, plays key role in the coagulation system and angiogenesis in tumors ${ }^{1-2}$. Fib imbalance is associated not only with coagulation malfunctions, which complicate coronary disorders and obesity, but also with some equally fatal diseases such as solid tumors and tumor metastases ${ }^{3-6}$. Systematic modification of Fib overproduction by anticoagulants and fibrinolytics was attempted some time ago ${ }^{7-9}$ and has recently been renewed to normal anticancer drugs ${ }^{9-11}$. During the past decade, molecular and genetic investigations have further revealed the key roles of Fib in cancer develop and possible mechanisms of action: for instance,
Fib-knockout mice showed greatly reduced tumor metastasis while the primary tumors were unaffected ${ }^{12}$, and some over-expression of Fib fragments has been demonstrated in mammary tumor cells in vitro ${ }^{13}$. These laboratory studies suggest that tumor cells also participate in Fib-related pathways and that Fib might be a potential therapeutic target. If this hypothesis is correct, studies of the basis of altered blood Fib concentrations (BFC) in cancer patients might be useful information of this kind more relevant than other general pathological information of human. Therefore, clinical BFC data from 139 cancer patients have been collected to offer a new insight to this hypothesis and stimulate further research of Fib-related pathogenesis and therapeutics. 


\section{MATERIALS AND METHODS}

One hundred and thirty nine adult cancer inpatients (aged 22- 82 years), enrolled in the Central Hospital of Jing-An District, Shanghai, China, were included in this survey. We received and enrolled patients according to the worldwide procedures of the Helsinki Agreements. The pathological characteristics of the patients were assessed by appropriate WHO classification protocols for clinico-pathological status (Table 1). Blood fibrinogen concentration (BFC) was determined for all patients both before and after one or two treatment regimens. Major treatments included surgery, chemotherapy and thermo-therapy, separately or in combination. The mean age of the male patients in this survey was 57.34 years (median 64 years) and the mean age of the female patients was 57.2 years (median 58.5 years).

Table 1: Characteristics of patients enrolled since 2002

\begin{tabular}{ll}
\hline Characteristics & No of patients \\
\hline Patients enrolled & 139 \\
Age range & Male (38-80), female (24-82) \\
Median & Male 65 years, female 58.5 years \\
Mean & Male 57.4 years, female 57.2 \\
& years \\
Pathological status (stages) & \\
T2 & 5 \\
T3 & 114 \\
T4 & 20 \\
Gender & \\
Male & 73 \\
Female & 66 \\
Race & \\
Chinese & All \\
Cancer latency & $0-20$ years \\
\hline
\end{tabular}

BFC was determined by a routine hospital protocol. Blood $(1.8 \mathrm{ml})$ was anticoagulated with sodium citrate $(3.8 \%, 0.2 \mathrm{ml})$. Mixed plasma was then taken to determine BFC using a STAGO Compact biochemical detector, manufactured in China.

\section{RESULTS}

To examine the hypothesis that there is a positive relationship between $\mathrm{BFC}$ and tumor-related pathological features, the following evidence was obtained. First, the mean BFC was $3.86(95 \%$ CI $1.24 \sim 6.48)$ and $4.34(95 \%$ CI $1.70 \sim 6.98) \mathrm{g} / \mathrm{L}$ in male and female patients respectively, about $35-50 \%$ above normal in approximately half of cancer cases (Table2). Secondly, the mean BFCs in patients with localized tumors were $4.02(95 \%$ CI $1.68 \sim 6.40) \mathrm{g} / \mathrm{L}(\mathrm{n}=6)$ (N0M0) and $4.08(95 \%$ CI $1.42 \sim 6.74) \mathrm{g} / \mathrm{L}(\mathrm{n}=26)(\mathrm{N} 1 \mathrm{M} 0)$, about
$5 \%$ lower than in those with long-distance metastases (N1M1), 4.22 (95\% CI 1.68 6.76) g/L: (n=107) (Table 4). Third, the mean BFC in patients varied with tumor type and origin. The value for lung cancer patients was $4.42(95 \%$ CI $1.68 \sim 7.16) \mathrm{g} / \mathrm{L}(\mathrm{n}=21)$, for hepatic cancers 4.36(95\% CI 1.96 6.74) $\mathrm{g} / \mathrm{L}(\mathrm{n}=5)$ and for stomach caners $4.63(95 \%$ CI $1.87 \sim 7.39) \mathrm{g} / \mathrm{L}(\mathrm{n}=8)$, all higher than the mean BFC in cancer patients as a whole 4.16 $\mathrm{g} / \mathrm{L}:(\mathrm{n}=139)$. In contrast, the mean $\mathrm{BFC}$ in patients with bowel or colon cancer was 3.79(95\% CI 1.35 6.23) $\mathrm{g} / \mathrm{L}(\mathrm{n}=16)$, which was lower than the overall mean of cancer patients (Table 5) but still higher than the value in healthy persons. Fourth, patients at different pathological stages varied in BFC level (Table 6). Patients in stage 3, which is the most aggressive and ongoing stage, had the highest average BFC levels.

Cancer patients tested both before and after chemotherapy, thermo-therapy or surgery showed a slight decrease in BFC, from $3.97(95 \%$ CI $1.67 \sim 6.27) \mathrm{g} / \mathrm{L}$ to $3.61(95 \%$ CI $1.65 \sim 5.57) \mathrm{g} / \mathrm{L}(\mathrm{n}=37)$. A persistent increase of BFC in patients treated by non-surgical therapy was observed, from $3.11(1.11 \sim 5.11)$ to $4.69(2.25 \sim 7.13) \mathrm{g} / \mathrm{L}(\mathrm{n}=17)$. Patients whose tumors had been removed by operation showed a dramatic decrease in mean BFC (from 4.70(95\% CI $2.46 \sim 6.94)$ to $3.23(95 \%$ CI $1.23 \sim 5.23) \mathrm{g} / \mathrm{L}, \mathrm{n}=20$; (Table 7). However, their BFC increased again with increasing time after surgery. For example, a woman patient with an initial BFC value of $3.8 \mathrm{~g} / \mathrm{L}$ showed a reduction to $2.17 \mathrm{~g} / \mathrm{L}$ in the second test, 2 weeks after surgery, and a subsequent increase to $3.61 \mathrm{~g} / \mathrm{L}$ at the third test.

We observed a difference in BFC as cancer latency increased. In patients with cancers of 0.5 to 1 years duration the value was $3.94(95 \%$ CI $1.32 \sim 6.56) \mathrm{g} / \mathrm{L}$; it was $4.15(95 \%$ CI $1.39 \sim 6.91)$ for 1 to 2 years, and highest in later stage patients (who survived longer than 2 years), 4.26(95\% CI 1.24 7.28) g/L (Table 8). Patients in the acute stage (diagnosed less than half a year previously) showed the highest BFC 4.51(95\% CI 1.43 7.59) $\mathrm{g} / \mathrm{L}$. All these data show that the level of BFC in cancer patients is linked to both primary tumor growth and long-distance metastases.

\section{DISCUSSION}

Fib and endothelial cells are accumulated and malformed in solid tumor stroma nourishing and supporting tumor tissue to further enlargement, which are controlled and regulated by the interplaying of coagulation enzymes and angiogenesis factors ${ }^{9-15}$. Methods for disrupting these pathological processes could have great clinical significance ${ }^{-11}$. Nowadays, present cancer chemotherapy is largely depending on empirical rather than well-targeted therapeutic. This often results losing the best opportunity of treatment in 
Online J. Biol. Sci., 7 (1): 8-11, 2007

Table 2: Comparison of plasma fibrinogen concentrations in normal subjects and cancer patients

\begin{tabular}{llll}
\hline Physical condition & Age years & \multicolumn{2}{l}{ Mean BFC (CI 95\%) g/L } \\
\cline { 2 - 4 } & Range & Male & female \\
\hline Normal & $35-44$ & $2.41(1.23 \sim 3.59)$ & $2.52(1.64 \sim 3.40)$ \\
Cancer & $24-82$ & $3.86(1.24 \sim 6.48)$ & $4.34(1.70 \sim 6.98)$ \\
\hline
\end{tabular}

Table 3: The blood fibrinogen concentrations in male and female cancer patients

\begin{tabular}{lllllll}
\hline Sex & Numbers & Age (years) & \multicolumn{2}{l}{ Mean fibrinogen, g/l } \\
\hline & & Median & Mean & Range & X $($ CI 95\%) & Higher Fib* \\
Male & 73 & 65 & 57.3 & $40-80$ & $3.86(1.24 \sim 6.48)$ & $20 / 26 \%$ \\
Female & 66 & 58.5 & 57.2 & $24-82$ & $4.34(1.70 \sim 6.98)$ & $19 / 29 \%$ \\
\hline
\end{tabular}

* Higher Fib—blood fibrinogen concentrations $>4.5 \mathrm{~g} / \mathrm{l}$; (positive for high fibrinogen concentration)

Table 4: The blood fibrinogen concentrations in cancer patients with different TNM

\begin{tabular}{lll}
\hline TNM & No of cases & Blood fibrinogen levels \\
& $\mathrm{n}$ & $\mathrm{X}(\mathrm{CI}$ 95\%) g/L \\
\hline N1M1 & 107 & $4.22(1.68 \sim 6.76)$ \\
N1M0 & 26 & $4.08(1.42 \sim 6.74)$ \\
N0M0 & 6 & $4.02(1.68 \sim 6.40)$ \\
\hline
\end{tabular}

Table 5: The blood fibrinogen concentrations in patients with tumors at different pathological stages

\begin{tabular}{lll}
\hline Stages & No of patients & $\begin{array}{l}\text { Blood fibrinogen levels } \\
\text { X }(\text { CI 95\%) g/L }\end{array}$ \\
\hline 2 & 5 & $3.30(1.72 \sim 4.88)$ \\
3 & 114 & $4.33(2.23 \sim 6.43)$ \\
4 & 20 & $3.53(1.79 \sim 5.27)$ \\
\hline
\end{tabular}

Table 6: The blood fibrinogen concentrations in patients with different tumors

\begin{tabular}{lll}
\hline Cancer origins & No of patients & Mean blood fibrinogen concentrations \\
\cline { 2 - 3 } & & $\mathrm{X}(\mathrm{CI}$ 95\%) g/L \\
\hline Pulmonary & 21 & $4.42(1.68 \sim 7.16)$ \\
Hepatic & 5 & $4.36(1.98 \sim 6.74)$ \\
Gastric & 8 & $4.63(1.87 \sim 7.39)$ \\
Bowel and colon & 16 & $3.79(1.35 \sim 6.23)$ \\
Total & 139 & $4.16(1.42 \sim 6.90)$ \\
\hline
\end{tabular}

Table 7: The changes of plasma fibrinogen level after one or several treatment regimens

\begin{tabular}{llll}
\hline No of patients & Operations & \multicolumn{3}{l}{ Blood fibrinogen concentrations g/L } & $2^{\text {nd }}$ test \\
$\mathrm{N}$ & & $4.70(2.46 \sim 6.94)$ & $3.23(1.23 \sim 5.23)$ \\
20 & Yes & $3.11(1.11 \sim 5.11)$ & $4.69(2.25 \sim 7.13)$ \\
17 & No & $3.97(1.67 \sim 6.27)$ & $3.61(1.65 \sim 5.57)$ \\
\hline
\end{tabular}

patients in both rapidity and correctness ${ }^{16}$. An improved individualized cancer therapy has been sought out by measurement of pathological profile of patients and other approaches ${ }^{16}$. BFC can be an indicator for launching more powerful individualized cancer chemotherapy. Since these pathological processes are highly complicated and currently not been fully understood, we need much more experiments to focusing and pinpointing them. BFC, as a key initiator of these multi-stage processes, was extensively measured in this study, especially observation of its level in different stages of tumor-related pathogenesis. 
Online J. Biol. Sci., 7 (1): 8-11, 2007

Table 8: The relation between blood fibrinogen concentrations and proposed cancer latency

\begin{tabular}{lll}
\hline $\begin{array}{l}\text { Patient numbers } \\
\mathrm{N}\end{array}$ & $\begin{array}{l}\text { Cancer latency } \\
\text { Years }\end{array}$ & $\begin{array}{l}\text { Blood fibrinogen levels } \\
\mathrm{X}(\mathrm{CI} 95 \%) \mathrm{g} / \mathrm{L}\end{array}$ \\
\hline 36 & $<0.5$ & $4.51(1.43 \sim 7.59)$ \\
46 & $0.5-1$ & $3.94(1.32 \sim 6.56)$ \\
32 & $1-2$ & $4.15(1.39 \sim 6.91)$ \\
21 & $>2$ & $4.26(1.24 \sim 7.28)$ \\
\hline
\end{tabular}

Although this work can not reach a clear-cut relation between Fib-related pathogenesis and drug sensitivity, it is a valuable original guideline for understanding tumor-Fib relation, especially useful in understanding the targeting and therapeutics-interaction of this kind and encouraging further systematic approaches. This study seems to reveal that Fib may be more closely related to stages and latency of tumors, tumor original tissue types whether or not a surgery is undergone but not only related to blood coagulation as previous reports. For the previous ones only provide information for anticoagulant or fibrinolytic treatments. The anticoagulant or fibrinolytic treatments have the side-effect of unbalancing the blood coagulation system $3,7,11$, especially if the use is in long-term ${ }^{3}$. Genetic investigation during the past decade suggests that tumor cells and metastases may arise from tumor Fib over-expression or assembly ${ }^{12-13}$. Given this information, we can determine the pharmacological mechanisms of anticancer drugs at each step and validate them clinically. Along with this investigation and previous data, we suggest a new individualized system for targeting tumor-Fib pathways may be applicable. Targeting these critical Fib-related steps with a new system of chemotherapy may confer beneficial effects, especially in combination with other chemotherapeutic regimens. Now anticancer drugs can also be selected as targeted therapy ${ }^{11}$. These data suggest that Fib levels can be valuable indicators of drug choices. To conclude, BFC can be a marker for choice targeted treatment and foresee the genetic status of tumor malignancy - as useful plasma data for selecting individualized cancer chemotherapy.

List of abbreviations used: Fib, fibrinogen; BFC, blood fibrinogen concentration

\section{REFERENCES}

1. Zacharski, L.R., 1981. Anticoagulation in the treatment of cancer in man. In: Donati MB, Davidson J.E., Garattini E, Ed.: Malignancy and hemostatic system. NY. Raven Press: pp: 113-128.
2. Staton, C.A., N.J. Brown and C.E. Lewis. 2003. The role of fibrinogen and related fragments in tumour angiogenesis and metastasis. Expert Opinion Biol. Therapy, 3: 1105-1120.

3. Kyrle, P.A., S. Eichinger, 2005. Deep vein thrombosis, Lancet, 365: 1163-1174.

4. Lam, T.H., L.J. Liu, E.D. Janus, C. Bourke and A.J. Hedley, 1999. The relationship between fibrinogen and other coronary heart disease risk factors in a Chinese population. Atherosclerosis, 143: 405-413.

5. Iyigun, I. and Y. Bakirci, 2002. Plasma concentrations of C-reactive protein and fibrinogen in ischaemic stroke. J. Int. Med. Res., 30: 591-596.

6. Bahia, L., L.G. Aguiar, N. Villela, D. Bottino, A.F. Godoy-Matos, B. Geloneze, M. Tambascia and E. Bouskela, 2006. Relationship between adipokines, inflammation, and vascular reactivity in lean controls and obese subjects with metabolic syndrome. Clinics, 61: 433-440.

7. Costantini, V. and L.R. Zacharski, 1992. The role of fibrin in tumor metastasis. Cancer Metastasis Rev. 11: 283-290.

8. Eikelboom, J.W. and S.R. Mehta, 2000. Venous thromboembolism and cancer. New Engl. J. Med. 343: 1337-1338.

9. Lu, D.Y., X.L. Chen and J. Ding, 2007. Treatment of solid tumors and metastases by fibrinogen-targeted anticancer drug therapy. Med. Hypotheses, 68:188-193.

10. Lu, D.Y., J.Y. Cao, Y.P. Huang, L. Gong,X.L. Chen, E.H. Chen and B. Xu, 1999. Comparison of some antineoplastic drugs on inhibiting thrombin catalyzing fibrinogen clotting in vitro. Chin. Med. J. 112: 1052-1053.

11. Lu, D.Y., J. Chi, L.P. Lin, M.Huang, B. Xu and J. Ding, 2004. Effects of anticancer drugs on the binding of ${ }^{125}$ I-fibrinogen to two leukemia cell lines in vitro. Int. J. Med. Res., 32: 488-491.

12. Palumbo, J.S., J.M. Potter, L.S. Kaplan, K. Talmage, D.G. Jackson and J.L. Degen, 2002. Spontaneous hematogenous and lymphatic metastasis, but not primary tumor growth or angiogenesis, is diminished in fibrinogen-deficient mice. Cancer Res., 62: 6966-6972.

13. Rybarczyk B.J. and P.J. Simpson-Haidaris, 2000. Fibrinogen assembly, secretion, and deposition into extracellular matrix by MCF-7 human breast carcinoma cells. Cancer Res. 60: 2033- 2039.

14. Browder, T., J. Folkman and S. Pirie-Shepherd, 2000. The hemostatic system as a regulator of angiogenesis. JBC. 275: 1521-1524.

15. Dvorak, H.F., 2003. How tumors make bad blood vessels and stroma. Am. J. Path. 162: 1747-1757.

16. Lu, D.Y., X.L. Chen and J.Ding, 2006. Individualized cancer chemotherapy, integrating drug sensitivity tests, pathological profile analysis and computational coordination-an effective strategy to improve clinical treatment. Med Hypotheses., 66: 45- 51. 